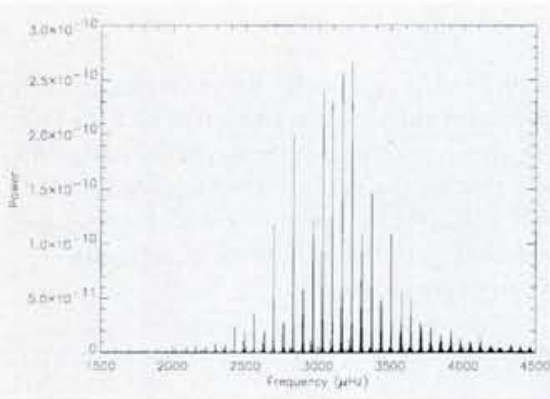

Fig. 5. Fourier-analysis of solar pressure oscillations ( $p$ modes), as observed by SOHO's VIRGO experiment, showing the spectrum in the region of the five-minute period. These data were obtained by an instrument observing the entire solar hemisphere facing Earth, i.e., by observing the Sun in the same way as a star.

First findings were then presented in a series of invited talks, equally shared between the two missions. Those on ISO covered stellar evolution and starformation in the Milky Way, the infrared emission from normal galaxies and ISO's observations of active and ultraluminous galaxies. SOHO reports covered the physical processes in the solar atmosphere, as seen through ultraviolet observations, new insights into corona and heliosphere through ultrasensitive observations in the optical domain, the diagnostic power of mass spectroscopy of the solar wind, and the structure and dynamics of the solar interior as revealed by helioseismology.

The figures illustrate highlights from the first results. One of the early surprises from ISO was the detection of an emission line from water vapour in the young planetary nebula, NGC 7027 (Fig. 1). This was unexpected as the object is carbon-rich and the oxygen was thought to be tied up in carbon dioxide rather than in water. Since then, ISO has detected many different emission lines from water vapour across most of its wavelength range in a variety of sources, including Herbig Haro objects and the variable star W Hydrae.

ISO's increased spatial resolution as compared to the earlier infrared sky survey made by the IRAS satellite is being demonstrated by a variety of spectacular maps. Examples include: maps (Fig. 2) at all ISO wavelengths of the resolved spiral galaxy M51, giving detailed information about the star formation underway in the spiral arms; and maps (Fig. 3) of the rho Ophiuchi dark cloud (which is completely obscured in the visible spectral region) at wavelengths of 7 and 15 microns showing a large number of young stars and regions so dense that they are opaque even to ISO.

$\mathrm{SOHO}$ has made measurements of temperature and flow velocity in the solar corona. The coronagraphs on SOHO have provided the first measurements in the region where the solar wind is accelerated. (The solar wind is the hot plasma that is released by the Sun - sometimes in steady streams, sometimes in gusts - and reaches the Earth after two or three days when it affects the geomagnetic environment.) The measurements show that the wind is accelerated from a few $\mathrm{km} / \mathrm{s}$ near the solar surface to hundreds of $\mathrm{km} / \mathrm{s}$ at $2.0-2.5$ solar radii. $\mathrm{SOHO}$ is also providing the first detailed view of the outer layers of the solar interior (Fig. 4). Data from the Michelson Doppler Imager instrument show that the uppermost convection cells (the so-called super-granule cells) are found to be flat and pancake-shaped. In the generally accepted mixing-length theory it was previously assumed that the cells were deep and shaped like spheres.

The participants were anxious to learn whether SOHO's helioseismology experiments had already yielded some hints about the structure of the innermost core of the Sun that could shed light on the neutrino problem. Existing helioseismology data, contrary to neutrino data, is well explained by the Standard Solar Model. Teo Roca-Cortés (Tenerife) reported that gravity modes (which carry information on the solar core) have not yet been seen. He expressed optimism though, since the

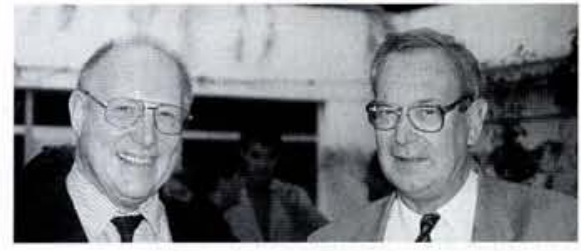

M.C.E. Huber, on the left, with G. Tammann from Basel who gave the plenary talk Expansion of the Universe.

noise level found in the SOHO oscillation data is more than 10-times lower than in those obtained from the ground.

An example of the oscillations spectrum around the five-minute period is given in Fig. 5. The spectrum shows eigenfrequencies of pressure modes which predominantly probe the outer layers of the Sun. While the "lines" at the lowfrequency end are extremely sharp, a significant broadening is noted at higher frequencies: apparently the lifetime of the high-frequency modes is shorter than that of low-frequency modes (3-4 days versus 34 months) and, accordingly, the frequency is more uncertain at high frequencies. Long observing periods will be needed to accurately determine the oscillation frequencies and line profiles, and to follow their change with the Sun's magnetic cycle. There also appears to be a hyperfine structure on high-frequency peaks that may be due to rotation below the convection zone.

\title{
Unique Opportunities with Storage Rings
}

R. Schuch and M. Larsson from Stockholm University report that the EPS-10 symposium Physics with Ion

Storage Cooler Rings demonstrated the unique opportunities offered by ion storage cooler rings.

With its high currents of cooled ions and cold electrons, the storage ring provides unique conditions for precision investigations in atomic, molecular, and nuclear physics. In reviewing the various cooling methods for stored ions with $\mathrm{MeV}$ kinetic energy (stochastic, electron and laser cooling), the EPS-10 symposium Physics with Ion Storage Cooler Rings emphasized recent developments, notably in electron cooling where meV electron temperatures are reached by adiabatically expanding the electron beam, first implemented at Stockholm's CRYRING.

Andreas Wolf (Heidelberg) described how electron coolers in storage rings serve two purposes: they act as cooling agents for the stored ion beam and as targets in electron-ion collisions. The electron and ion beams move with the same average velocity when the ion beam is phase-space cooled. If the electron-beam velocity is changed so that the electrons move faster (or slower) than the ions, electron-ion collisions at a well-defined energy down to the meV range are induced.

Collisions between electrons and atomic ions lead to recombination if the free electron can deposit its energy to the ion while being captured, and if the capture process can be stabilized. For a bare ion containing no electrons, capture and stabilization occurs in one step by the

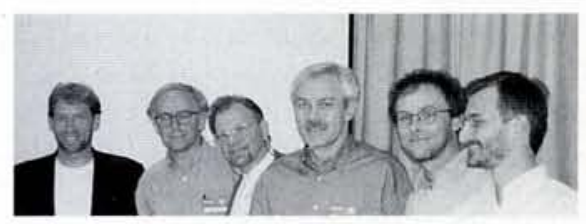

The speakers and organizers of the EPS-10 symposium Physics with lon Storage Cooler Rings From left to right: J. Hangst (Aarhus), M. Larsson (Stockholm), J. Eades (CERN), R. Schuch (Stockholm), T. Stöhlker (Darmstadt), and A. Wolf(Hedielberg). 
emission of a photon. If the ion carries bound electrons, the excess energy of the free electron is removed by excitation of a bound electron. Emission of a photon stabilizes the capture process and dielectronic recombination is completed.

Research groups at European ionstorage rings collaborate within the framework of a European Union-funded network. Electron-ion interactions are being studied with the electron coolers at those rings which have atomic physics programmes, namely ASTRID (Aarhus University), CRYRING, ESR (GSI, Darmstadt), and TSR (Max-Planck Institute for Nuclear Physics, Heidelberg). One interesting aspect of dielectronic recombination described by Wolf is the effect of electric fields. Experiments with $\mathrm{Si}^{\mathrm{H+}}$ to address this issue used a transverse magnetic field introduced in the CRYRING electron cooler. The stored ion beam was misaligned at different angles with respect to the field to introduce a motional electric field of controlled strength. Dielectronic recombination spectra recorded up to field strengths close to $200 \mathrm{~V} / \mathrm{cm}$ showed that the electric field had a large effect on recombination into high Rydberg states, whereas low Rydberg states were relatively unaffected. Theoretical work is underway to try to understand the measured spectra. Wolf also described how molecular ions can recombine by means of dissociation, and how imaging experiments at storage rings can determine neutral atomic states into which the molecule dissociates. The high luminosity available in ion storage rings have made possible a new generation of experiments that were previously impossible.

In contrast to laser cooling in traps, laser cooling in storage rings poses particular problems (Jeff Hangst, Aarhus). The lasers can be merged collinearly with the stored ion beam to facilitate cooling in the longitudinal direction. However, the beam is not cooled directly in the transverse direction. Hence the extreme temperatures obtained in neutral traps are not available in storage rings. Nevertheless, temperatures on the order of $1 \mathrm{mK}$ must be considered as very low in connection with fast beams. An interesting aspect of ion-beam dynamics is that the ground state is still unknown. A Schottky spectrum of many uncorrelated particles shows a broadening which reflects the momentum spread in the beam. When laser cooling is applied, the temperature and particle density reach a regime where the particles can no longer be considered as uncorrelated. Coherent

\section{A Tale of Two (Novel) Atoms}

A highlight of the EPS-10 symposium Physics with Ion Storage Cooler Rings was a report by John Eades (CERN) on the production of antihydrogen and antiprotonic helium at CERN's Low Energy Antiproton Ring (LEAR). High-precision spectroscopic studies (see figure) of antiprotomic helium showing that metastable states are responsible for the exceptional long lifetime are already well advanced. He discussed plans to study CPT invariance and the gravitational properties of antimatter using antihydrogen and antihelium. Both promise to become benchmark atoms for the study of fundamental matter — antimatter symmetries.
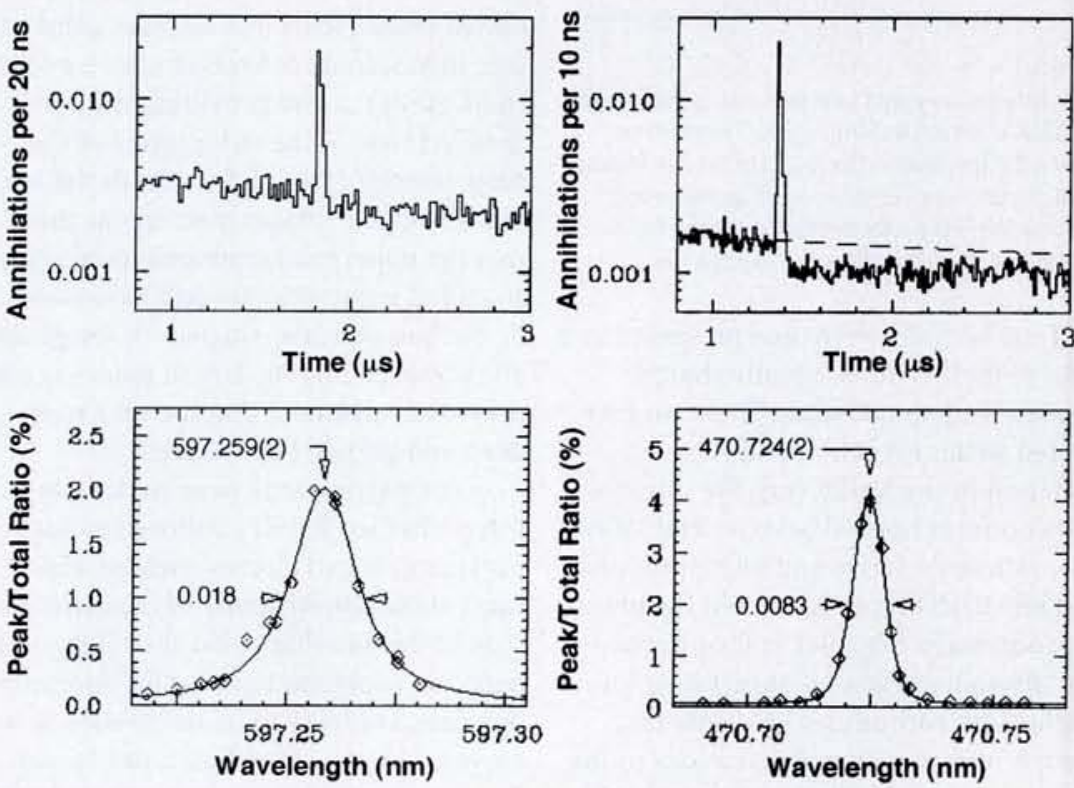

Two examples of laser-induced transitions in metastable antiprotonic helium atoms. Left: From $(n, L)=(39,35)$ to $(n, L)=(38,34)$; Right: From $(n, L)=(37,34)$ to $(n, L)=$ $(36,33)$. These transitions belong to the sequences with vibrational quantum number $v(=n-L+1)$ equal to 3 and 2 , respectively. The upper panels show the spectrum of time delays between the antiproton's capture by a helium atom and its subsequent annihilation, the sharp peaks being forced by the resonance of the laser

components in the Schottky spectrum of laser-cooled $\mathrm{Mg}^{+}$have been observed at ASTRID. At TSR, transverse cooling arising from transverse - longitudinal coupling by intrabeam scattering has been observed for $\mathrm{Be}^{+}$. Thus, "liquid beams" have been produced; it remains to be seen if they also crystallize.

Thomas Stöhlker's (GSI, Darmstadt) overview of atomic-physics experiments addressing QED effects in hydrogen-like high- $Z$ ions gave, as a representative example, the is binding energy in H-like uranium which could be determined with an absolute precision of $16 \mathrm{eV}$. This is up to now the most precise value available for the high- $Z$ regime. Also discussed was hyperfine splitting of the ground state in $\mathrm{H}$-like bismuth measured very accurately by collinear laser spectroscopy. GSI's ESR storage ring has also proven to be a powerful tool for determining the ground-state properties of nuclei. Owing to the well- pulse (at a fixed time) on the transition frequencies. The lower panels show the area of this peak as the laser wavelength is scanned through the resonance. The $v=$ 2 resonance clearly shows that the forced annihilations are accompanied by a depletion of the annihilation spectrum at later times. The rate at which the depleted spectrum recovers after the laser pulse gives valuable information on the population and lifetime of the initial state levels.

defined velocity of the circulating ions, provided by the electron cooler, the ESR acts as a precise and effective mass spectrometer which can be exploited by a sensitive analysis of the revolution frequency of the stored ions ("Schottky analysis"). The resolving power is demonstrated by the mass-resolved ground and isomeric states of ${ }^{52} \mathrm{Mn}^{25+}$ which are separated by only $370 \mathrm{keV}$. The beta-lifetimes of highlycharged ions can be also be addressed by applying the same Schottky technique. In particular, exotic weak decay modes can be studied, e.g., the bound-state beta decay which is an important decay channel in hot stellar plasmas during nucleosynthesis. An interesting example is the lifetime of bare ${ }^{187} \mathrm{Re}^{75+}$ which, due to the bound beta-decay, is nine orders of magnitudes shorter than that of neutral ${ }^{187} \mathrm{Re}$. This result is of particularly relevance since ${ }^{187} \mathrm{Re}$ serves as one of the few clocks for the age of the universe. 